Series A

I. MATHEMATICA

$336 / 15$

\title{
ÜBER DAS NORMALENBÜNDEL DIFFERENZIERBARER MANNIGFALTIGKEITEN
}

voN

K. P. GROTEMEYER 
Am 13. September 1963 vorgelegt von P. J. Myrberg und Oldi Lehto 


\section{Über das Normalenbündel differenzierbarer Mannigfaltigkeiten}

In dieser Note sollen einige Fragestellungen der globalen Differentialgeometrie behandelt werden, die zum Teil schon mehrfach Gegenstand von Betrachtungen waren und zum Teil neu sind. Hier soll in erster Linie von kompakten Mannigfaltigkeiten im $\boldsymbol{R}^{N}$ die Rede sein. Später sogar nur von Flächen in $\boldsymbol{R}^{3}$. Es sei $M^{n}$ eine differenzierbare Mannigfaltigkeit. (Die differenzierbare Struktur werde repräsentiert durch einen $C^{\infty}$-Atlas.)

Eine differenzierbare Abbildung $\mathfrak{x}: M^{n} \rightarrow \boldsymbol{R}^{N}, \quad N \geqq n$, wobei $\mathfrak{x}$ in jedem Punkt maximalen Rang besitzt, d. h. in jeder Karte sind die partiellen Ableitungen $\mathfrak{x}_{1}, \ldots, \mathfrak{x}_{n}$ linear unabhängig, heisst Immersion. Ist überdies $\mathfrak{x}$ injektiv und topologisch, so spricht man von einer Einbettung.

1. Das Normalenbündel. In jedem Punkt von $\mathfrak{x}\left[M^{n}\right]$ bilden die Normalen einen $\boldsymbol{R}$-Vektorraum der Dimension $N-n$. Die disjunkte Vereinigung dieser Vektorräume, in natürlicher Weise versehen mit einer Topologie, kann als »Bündelraum» eines Vektorraumbündels über $\mathfrak{x}\left[M^{n}\right]$ (oder auch $M^{n}$ ) als Basisraum aufgefasst werden. Die Faser über einem Punkt ist gerade der Normalenraum an dieser Stelle. Strukturgruppe ist die GL $(N-n, \boldsymbol{R})$. Wir sprechen kurz vom Normalenbündel $\left.v_{\mathfrak{x}}{ }^{1}\right)$.

Ist der Basisraum parakompakt, so gestattet dieses Bündel eine Reduktion der Strukturgruppe auf die $\mathbf{O}(N-n)$, und man erhält aus dem $v_{x}$ ein $(N-n-1)$-Sphärenbündel, d. h. die Fasern sind $(N-n-1)$-dimensionale Sphären. Geometrisch bedeutet die Reduktion nichts anderes, als die Normierung aller vom Nullvektor verschiedenen Normalenvektoren. Der Bündelraum, wir bezeichnen ihn mit $B$, dieses Bündels aller normierten Normalenvektoren trägt selbst die Struktur einer $(N-1)$-dimensionalen differenzierbaren Mannigfaltigkeit. (Einen Atlas von $B$ erhält man durch die kartesischen Produkte der Karten von $M^{n}$ und $S^{N-n-1}$.)

$\left.{ }^{1}\right)$ Ist $\mathfrak{x}$ eine Einbettung und $M^{n}$ kompakt und orientiert, so verschwindet die Eulersche charakteristische Cohomologieklasse $\mathfrak{X}\left(\nu_{\mathfrak{x}}\right) \in H^{n}\left(M^{n}, Z\right)$ des Normalenbündels $v_{\mathfrak{x}}$. Ist $\mathfrak{x}$ eine Immersion, so ist das im allgemeinen nicht der Fall. Hierdurch wird die Bedeutung der Normalenbündel für Einbettungsfragen beleuchtet. Vgl. etwa [14]. 
A. I. $336 / 15$

2. Die sphärische Abbildung. Es ist nun naheliegend, die Gausssche sphärische Abbildung zu übertragen: $\mathfrak{x}\left[M^{n}\right] \subset R^{N}$; sei $S^{N-1}$ die Einheitssphäre im $\boldsymbol{R}^{N}$. Ist $\mathfrak{n} \in B$, so sei $\sigma(\mathfrak{n})$ der dem Einheitsvektor $\mathfrak{n}$ entsprechende Punkt von $S^{N-1}$, der $\mathfrak{n}$ als Ortsvektor besitzt. Offenbar ist dann

$$
\sigma: \quad B \rightarrow S^{N-1}
$$

eine differenzierbare Abbildung (von der Klasse $C^{r-1}$, wenn $\mathfrak{x}$ von der Klasse $C^{r}$ ist). Man nennt $\sigma$ die sphärische Abbildung von $\mathfrak{x}\left[M^{n}\right]$ und $\sigma[B]$ heisst sphärisches Bild.

Aufgrund des Theorems von Sard [13] ist die Bildmenge unter $\sigma$ derjenigen Punkte von $B$, in denen $\sigma$ singulär wird (d.h. in denen Rang $\sigma$ $<N-1)$ auf $S^{N-1}$ vom Masse Null.

Bezeichnet $d \omega$ das Volumenelement von $S^{N-1}$ und ist

$$
c_{N-1}=\int d \omega,
$$

so stellen

$$
\frac{1}{c_{N-1}} \int^{\sigma[B]} d \omega
$$

und

$$
\frac{1}{c_{N-1}} \int^{\sigma[B]}|d \omega|
$$

den normierten bzw. den normierten absoluten Inhalt des sphärischen Bildes dar. Ist $\sigma_{*}$ die durch $\sigma$ induzierte "duale" Abbildung, so erhalten die beiden Integrale die Form:

$$
\begin{aligned}
& I(\mathfrak{x}, n, N)=\frac{1}{c_{N-1}} \int^{B} \sigma_{*}(d \omega), \\
& T(\mathfrak{x}, n, N)=\frac{1}{c_{N-1}} \int^{B}\left|\sigma_{*}(d \omega)\right| .
\end{aligned}
$$

Speziell für Kurven und Flächen im $\boldsymbol{R}^{\mathbf{3}}$ gilt:

$$
\begin{aligned}
& T(\mathfrak{x}, 1,3)=\frac{1}{\pi} \int|k| d s, \\
& T(\mathfrak{x}, 2,3)=\frac{1}{2 \pi} \iint|K| d o,
\end{aligned}
$$


wobei $k$ die Krümmung der Kurve, $K$ die Gausssche Krümmung der Fläche, $d s$ das Bogenelement und $d o$ das Oberflächenelement von Kurve bzw. Fläche ist. Analoge Formeln ohne Absolutstriche gelten für die Integrale $I(\mathfrak{x}, 1,3), I(\mathfrak{x}, 2,3)$.

3. Das Problem von Chern, Lashof, Kuiper für die Totalkrümmung $T(\mathfrak{x}, n, N)$. [1], [2], [10]. Aus der Formel von Gauss-Bonnet kann man entnehmen, dass $T(\mathfrak{x}, 2,3) \geqq 2$ für kompakte Flächen gilt. Nach Fenchel [4] gilt Entsprechendes auch für geschlossene Kurven: $T(\mathfrak{x}, 1,3) \geqq 2$.

Allgemein vermutet man daher für kompakte $M^{n}$ :

$$
T(\mathfrak{x}, n, N) \geqq 2 .
$$

Chern und Lashof [1] und unabhängig Kuiper [10] haben das bestätigt. Genauer gilt sogar:

$$
T(\mathfrak{x}, n, N) \geqq \sum_{k=0}^{n} \beta_{k},
$$

wobei $\beta_{k}=\operatorname{Rang} H_{k}\left(\mathfrak{x}\left[M^{n}\right], R\right)$ die $k$-te Bettizahl ist. Da $\beta_{0}=$ $\beta_{n}=1$, so folgt aus (2) sofort auch (1).

Die Diskussionen des Falles der Gleichheit finden sich in [2] und [10].

Die Beweisidee von (2) beruht auf der Theorie von Morse.

Man »zählt» mit ihrer Hilfe, wie oft ein Punkt der Sphäre $S^{N-1}$ als Funktionswert unter $\sigma$ auftritt. In der Funktion

$$
z: \quad \mathfrak{x}\left[M^{n}\right] \rightarrow \boldsymbol{R}
$$

definiert durch $z=\langle\mathfrak{n} \mathfrak{x}\rangle$, wobei $\mathfrak{n} \in S^{N-1}$ beliebig aber festgewählt sei und die Klammern das Skalarprodukt in $R^{N}$ bedeuten, besitzt man eine solche, die gerade dort kritische Stellen besitzt, in denen $\mathfrak{n}$ normal ist $\mathrm{zu} \mathfrak{x}\left[M^{n}\right]$. (Dort ist $z_{i}=0, \quad i=1,2, \ldots, n$.) Eine solche kritische Stelle heisst ausgeartet, wenn die Determinante der zweiten partiellen Ableitungen verschwindet: Det $\left(z_{i \mid k}\right)=0, d . h$. wenn die sphärische Abbildung $\sigma$ gerade singulär ist. Nach dem in 1 Bemerkten, kann die Menge derartiger $\mathfrak{n} \in S^{N-1}$ höchstens das Mass Null besitzen. Zu fast allen $\mathfrak{n}$ gehören also nicht ausgeartete kritische Stellen. Aufgrund der Theorie von Morse ist für eine Funktion $z$ mit lauter nicht ausgearteten kritischen Stellen deren Gesamtzahl nicht kleiner als $\sum_{k=0}^{n} \beta_{k}$. Das heisst aber, dass fast jedes $\mathfrak{n} \in S^{N-1}$ mindestens $\left(\sum_{k=0}^{n} \beta_{k}\right)$-mal angenommen wird unter $\sigma$. Es gilt somit (2).

4. Eine Darstellung der Eulerschen Charakteristik. Der Hauptgedanke des vorstehenden Beweises liegt in dem Heranziehen der Funktion $z=$ 
$\langle\mathfrak{n} \mathfrak{x}\rangle$ und Anwendung der Theorie von Morse. Man kann nun auch ziemlich einfach eine Formel für $I(\mathfrak{x}, n, N)$ herleiten, wobei man sich analoger Schlussweisen bedient. Eine kritische Stelle heisse vom Typ $k$, wenn die Matrix der zweiten partiellen Ableitungen der Funktion in diesem Punkt genau $k$ negative Eigenwerte besitzt. Ist $\gamma_{k}$ die Gesamtzahl der kritischen Stellen vom Typ $k$ der betrachteten Funktion, so gilt $\gamma_{k} \geqq \beta_{k}$. (Daraus folgt die oben schon benutzte Ungleichung für die Gesamtzahl kritischer Stellen.) Besitzt die betrachtete Funktion nicht ausgeartete kritische Stellen, so gilt weiter

$$
\sum_{k=0}^{n}(-1)^{k} \gamma_{k}=\chi
$$

Dabei ist

$$
\chi=\sum_{i=0}^{n}(-1)^{i} \beta_{i}
$$

die Eulersche Charakteristik der Mannigfaltigkeit.

Nun sei

$$
\varrho: \quad S^{N-1} \rightarrow P^{N-1}
$$

die natürliche Abbildung der $S^{N-1}$ in den reellen projektiven Raum $P^{N-1}$, der durch Identifizierung von Diametralpunkten aus der $S^{N-1}$ entstanden ist. Weiter setzt $\operatorname{man} \tau=\varrho \circ \sigma, \mathrm{d} . \mathrm{h}$.

$$
\tau: \quad B \rightarrow P^{N-1} .
$$

An die Stelle der sphärischen Abbildung $\sigma$ lassen wir also $\tau$ treten. Es ist dann

$$
\int d \hat{\omega}=\frac{1}{2} \int^{P^{N-1}} d \omega=\frac{1}{2} c_{N-1},
$$

wenn $d \hat{\omega}$ das Volumenelement vom $P^{N-1}$ ist.

Nun soll bestimmt werden, wie oft $\mathfrak{n} \in S^{N-1}$ im »algebraischen Sinne» angenommen wird. Da die kritischen Stellen der Funktionen $\langle\mathfrak{n} \mathfrak{x}\rangle$ und $\langle(-\mathfrak{n}) \mathfrak{x}\rangle$ dieselben sind, betrachtet man besser anstatt der $S^{N-1}$ den $P^{N-1}$; denn hier stellen $\mathfrak{n}$ und $-\mathfrak{n}$ denselben Punkt dar. Lokal lässt sich jedes Element aus $B$ beschreiben als geordnetes Paar $\left(\mathfrak{n}, \mathfrak{x}_{0}\right)$, wobei $\mathfrak{n}$ ein normierter Normalenvektor im Punkte $\mathfrak{x}_{0}$ von $\mathfrak{x}\left[M^{n}\right]$ ist. Die Funktion $\langle\mathfrak{n} \mathfrak{x}\rangle$ besitzt in $\mathfrak{x}_{0}$ dann sicher eine kritische Stelle. Ist diese vom Typ $k$, so gilt: $\operatorname{sign}\left\{\tau_{*}(d \hat{\omega})\right\}=(-1)^{k}$. Wegen (3) werden fast alle Punkte von $P^{N-1} \quad \chi$-mal im algebraischen Sinne angenommen. Also gilt jetzt 


$$
\chi=\frac{2}{c_{N-1}} \int^{B} \tau_{*}(d \hat{\omega})
$$

und auch

$$
I(\mathfrak{x}, n, N)=\chi\left(\mathfrak{x}\left[M^{n}\right]\right) .
$$

5. Die Funktion $z=\langle\mathfrak{n} \mathfrak{x}\rangle$ im Fall der Flächen im $\boldsymbol{R}^{\mathbf{3}}$. Es ist nach dem Vorausgegangenen klar, dass die Funktion $z=\langle\mathfrak{n} \mathfrak{x}\rangle$ eine wesentliche Rolle bei den Betrachtungen gespielt hat. Daher mag es gestattet sein, diese Funktion auch im Rahmen der »Flächentheorie» zu studieren. Zunächst ziehen wir eine Integralformel für Vektorfelder auf Riemannschen Mannigfaltigkeiten heran:

Es sei $M$ eine zweidimensionale orientierte Riemannsche Mannigfaltigkeit, deren Masstensor $g_{i k}$ zur Klasse $C^{2}$ gehört.

$G \subset M$ sei ein schlichtes Gebiet auf $M$ mit kompakter abgeschlossener Hülle $G^{-}$. Der Rand $\partial G$ sei stückweise zur Klasse $C^{2}$ gehörig und so orientiert, dass die intrinseke Normale $n_{i}$ in jedem Randpunkt in das Innere von $G$ weist. Nun sei $\mu_{i}$ ein auf $G^{-}$definiertes Vektorfeld, das in $G$ zur Klasse $C^{2}$ gehört und auf $\partial G$ nach der Bogenlänge differenzierbar ist. Es gilt dann die Integralformel:

$$
\iint \frac{\left\|\mu_{i \| k}\right\|}{g} d o=\frac{1}{2} \int^{\partial G} \mu_{i} \mu^{i}(c+\dot{\omega}) d s+\frac{1}{2} \int^{G} \int k g^{i k} \mu_{i} \mu_{k} d o .
$$

Darin bedeutet $\mu_{i \| k}$ die kovariante Ableitung von $\mu_{i}$ nach der $k$-ten Koordinate und $\left\|g_{i k}\right\|=g$ die Determinante des Masstensors. $\omega$ ist in denjenigen Punkten von $\partial G$, in denen $\mu_{i} \neq 0$ ist, der Winkel zwischen Kurve und Vektorfeld, ds bezeichnet das Bogenelement und do das Oberflächenelement. $c$ ist die geodätische und $K$ die Gausssche Krümmung.

Der einfache Beweis der Formel (5) wird mit anderen Anwendungen an anderer Stelle erscheinen. Er beruht im wesentlichen auf der Integrabilitätsbedingung:

$$
\varepsilon^{s k} \mu_{i\|k\| s}=K \varepsilon_{i .}^{j} \mu_{j}
$$

und geeigneten Umformungen des Randintegrals. Als sehr einfache Anwendung erhält man aus (5) die Integralformel von Gauss-Bonnet ([11] S. 165 f.).

Sei jetzt $G$ ein Flächenstück im $R^{3}$. Dann betrachten wir das Vektorfeld $z_{i}=\left\langle\mathfrak{n} \mathfrak{x}_{i}\right\rangle$ und erhalten aufgrund der Ableitungsgleichungen 
$\mathfrak{x}_{i \| k}=B_{i k} \xi$, wenn $B_{i k}$ der Tensor der 2. Fundamentalform ist und $\xi$ die Flächennormale ${ }^{2}$ ), wegen

$$
\frac{\left\|z_{i \| k}\right\|}{g}=K\langle\mathfrak{n} \xi\rangle^{2}
$$

die Formel

$$
\iint K\left\{3\langle\mathfrak{n} \xi\rangle^{2}-1\right\} d o=\int^{\partial G} \nabla(z, z)(c+\dot{\omega}) d s .
$$

Darin ist $\nabla(z, z)=g^{i k} z_{i} z_{k}$ der erste Beltrami-Operator.

Für eine geschlossene Fläche $F$ gilt danach

$$
\iint K\langle\mathfrak{n} \xi\rangle^{2} d o=\frac{2 \pi}{3} \chi(F),
$$

worin $\chi(F)$ die Eulersche Charakteristik ist.

Das ist ein einfaches Analogon zur Formel über die curvatura integra. Allerdings ist der Integrand in $\left(6^{\prime}\right)$ von der Einbettung abhängig.

Bezeichnet

$$
H=\frac{1}{2} g^{i k} B_{i k}
$$

die mittlere Krümmung von $F$, so gilt weiter

$$
\iint\left(H^{2}-K\right)\langle\mathfrak{H} \xi\rangle^{2} d o \geqq \frac{2 \pi}{3}(2-\chi(F)),
$$

wobei die Gleichheit genau im Fall der Sphäre eintritt.

Zum Beweis zerlegt man $F=F_{+} \cup F_{-}$, dabei besteht $F_{+}$aus allen Punkten von $F$ in denen $K \geqq 0$ gilt, $F_{-}$besteht aus den übrigen, d. h. dort gilt $K<0$. Weiter gilt stets $H^{2}-K \geqq 0$. Man erkennt nun weiter

$$
\iint K\langle\mathfrak{n} \xi\rangle^{2} d o \geqq \iint\langle\mathfrak{n} \xi\rangle^{2} d \omega=\frac{4 \pi}{3} .
$$

Denn das sphärische Bild von $F_{+}$überdeckt die gesamte Sphäre $S^{2}$, da es zu jeder Richtung $\mathfrak{a} \neq \mathfrak{v}$ eine Stützebene an $F$ mit diesem Stellungsvektor gibt, die wegen der Differenzierbarkeit von $F$ Tangen-

2) Wegen der Bezeichnungsweise sei auf [5] oder [3] S. 149 ff. verwiesen. 
tialebene in einem Punkt mit $K \geqq 0$ sein muss. Wir finden aus (8) dann unter Verwendung von $\left.\left(6^{\prime}\right)^{3}\right)$

$$
\int^{F_{-}} \int(-K)\langle\mathfrak{n} \xi\rangle^{2} d o \geqq \frac{2 \pi}{3}(2-\chi(F)) .
$$

Auf $F_{-}$gilt nun weiter $H^{2}-K \geqq-K$. Somit

$$
\iint\left(H^{2}-K\right)\langle\mathfrak{n} \xi\rangle^{2} d o \geqq \iint(-K)\langle\mathfrak{n} \xi\rangle^{2} d o \geqq \frac{2 \pi}{3}(2-\chi(F)) .
$$

Gilt die Gleichheit, so muss

$$
\iint\left(H^{2}-K\right)\langle\mathfrak{n} \xi\rangle^{2} d o=0
$$

gelten, woraus $H^{2}-K \equiv 0$ auf $F_{+}$folgt. Dann ist $F_{+}$Stück einer Sphäre oder einer Ebene und $K=$ const $\geqq 0$ auf $F_{+}$. Da auf $F_{-}$sicher $K<0$ gilt, so muss aus Stetigkeitsgründen $F_{-}=\varnothing$ sein. D. h. $F$ ist eine Sphäre. Man kann daraus weiter eine Kennzeichnung der Sphären im $\boldsymbol{R}^{3}$ gewinnen. Bezeichnen wir mit $\triangle$ den zweiten Beltrami-Operator $\left(\triangle z=g^{i k} z_{i \| k}\right)$, so gilt der Satz:

Für jede geschlossene Fläche $F$ der Klasse $C^{3}$ im $\boldsymbol{R}^{3}$ gilt

$$
\iint(\triangle z)^{2} d o \geqq \frac{16}{3} \pi \text {. }
$$

Die Gleichheit tritt nur im Fall der Sphäre ein.

Zum Beweis hat man lediglich zu beachten, dass $\triangle z=2 H\langle\mathfrak{n} \xi\rangle$ gilt, was man unmittelbar aus $z_{i \| k}=B_{i k}\langle\mathfrak{n} \xi\rangle$ durch Überschieben mit $g^{i k}$ erhält. Nach (7) und $\left(6^{\prime}\right)$ ist nun

$$
\iint\left(\frac{\triangle z}{2}\right)^{2} d o=\iint H^{2}\langle\mathfrak{n} \xi\rangle^{2} d o \geqq \frac{2 \pi}{3}(2-\chi(F))+\frac{2 \pi}{3} \chi(F)=\frac{4 \pi}{3} .
$$

$\mathrm{Da}$ in (7) die Gleichheit nur für Sphären eintritt, so ist alles bewiesen.

6. Flächen mit gleichem sphärischen Bild. In diesem Abschnitt soll kurz eine schon häufig behandelte Fragestellung kurz beleuchtet werden ${ }^{4}$ ). Sei $M^{2}$ eine 2-dimensionale differenzierbare Mannigfaltigkeit, $\mathfrak{x}, \hat{\mathfrak{x}}$

3) Man erhält aus (8), (9) auch sofort die Formel

$$
\iint|K|\langle\mathfrak{n} \xi\rangle^{2} d o \geqq \frac{\pi}{3}(4-\chi(F)),
$$

wobei die Gleichheit nur für die Aleksandrowschen $T$-Flächen eintritt. [3] S. 39.

4) Vgl. etwa [6] und [9]. 
zwei Einbettungen in den $\boldsymbol{R}^{3}$. Wir haben dann das kommutative Diagramm:

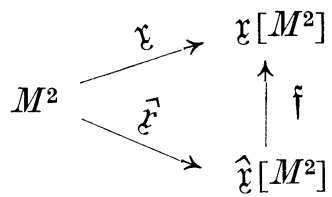

Bekannte Fragestellungen der globalen Flächentheorie entstehen dann, wenn man von $\mathfrak{x}$ und $\hat{\mathfrak{x}}$ gewisse Eigenschaften fordert und fragt, ob dann $\mathfrak{f}$ eine Bewegung ist. (Soll in entsprechenden Punkten $g_{i k}=$ $\hat{g}_{i k} \quad$ sein, so entsteht das Kongruenzproblem isometrischer Flächen.) Wir betrachten hier einen anderen Fall, nämlich den, dass $\xi=\hat{\xi}$ gilt. Es sollen also in entsprechenden Punkten die Normalen übereinstimmen. Wie man sich sofort überzeugt, ist diese Bedingung im allgemeinen keineswegs ausreichend, um irgendetwas über $\mathfrak{f}$ aussagen zu können. Beispielsweise lassen sich zwei Eiflächen stets in der angegebenen Weise durch "parallele Normalen» aufeinander abbilden. (Es gilt das für alle Flächen mit dem gleichen sphärischen Bild.) Es müssen also weitere Bedingungen gestellt werden. Üblich ist nun seit Christoffel und Minkowski (siehe [6]) das Gleichsein gewisser Krümmungsfunktionen (Summe bzw. Produkt der Hauptkrümmungsradien) in den entsprechenden Punkten zu verlangen. Nach A. D. Aleksandrow [6], [9] kann man auch zum gleichen Resultat kommen, wenn man verlangt, dass eine Funktion, die gewisse Eigenschaft hat, der Krümmungsfunktionen für beide Flächen in entsprechenden Punkten gleiche Werte besitzt. Bei diesen Funktionen handelte es sich um solche, die in den Variablen gleichsinnig monoton sind. Wie sich herausstellt, ist es sogar für die Betrachtungen überflüssig, eine funktionale Bindung für die Krümmungsfunktionen vorzusehen. Es genügen gewisse Ungleichungen, gewissermassen als Ersatz für die Monotoniebedingungen.

Geben wir noch einen kurzen Einblick in die beiden wichtigsten Untersuchungsmethoden für den angeschnittenen Problemkreis. Weil $\mathfrak{x}$ und $\hat{\mathfrak{x}}$ in entsprechenden Punkten parallele Normalen besitzen, so gilt für $\mathfrak{f}=\mathfrak{x}-\hat{\mathfrak{x}}$

$$
\mathfrak{f}_{i}=\pi_{i}^{k} \mathfrak{x}_{k} .
$$

Ziel ist der Nachweis, dass beide Flächen translationsgleich sind, d. h. $\mathfrak{f}=$ const. Statt $\pi_{i}^{k}$. betrachtet man lieber den symmetrischen Tensor $\sigma_{i k}=\pi_{i}^{s} B_{s k}$. Wenn man sich auf Flächenstücke mit positiver Gaussscher Krümmung beschränkt, so kann man zwei positiv definite Tensoren $P^{i k}, Q^{i k}$ angeben, so dass gilt

$$
\begin{aligned}
& P^{i k} \sigma_{i k}=R_{1}+R_{2}-\left(\hat{R}_{1}+\hat{R}_{2}\right), \\
& Q^{i k} \sigma_{i k}=R_{1} R_{2}-\hat{R}_{1} \hat{R}_{2} .
\end{aligned}
$$


Darin sind $R_{1}, R_{2}$ bzw. $\hat{R}_{1}, \hat{R}_{2}$ die Hauptkrümmungsradien von $\mathfrak{x}$ bzw. $\hat{\mathfrak{x}}$. Elementar lässt sich nun zeigen: Wenn in jedem Punkt stets $\left(P^{i k} \sigma_{i k}\right)\left(Q^{\alpha \beta} \sigma_{\alpha \beta}\right) \leqq 0$ gilt, d. h. wenn die beiden Funktionen niemals gleiches Vorzeihen besitzen, dann gilt $\left\|\pi_{i .}^{k}\right\| \leqq 0$ und aus $\left\|\pi_{i}{ }^{k}\right\|=0$ folgt $\pi_{i .}^{k}=0$.

Aufgrund dieses Hilfssatzes kommt es darauf an zu zeigen, dass $\left\|\pi_{i}^{k}\right\|=0$ gilt. Das kann auf zwei verschiedene Weisen geschehen:

a) Die Integralformelmethode [6]. Man verschafft sich eine Integralformel, in der in einem Oberflächenintegral $\left\|\pi_{i}^{k}{ }^{k}\right\|$ auftritt:

$$
\int^{G} \int\left\|\pi_{i .}^{k}\right\| A d o=\int^{\partial G} \ldots+\int^{G} \int \ldots
$$

Oftmals gelingt es zu zeigen, dass der mit $A$ bezeichnete Ausdruck ein festes Vorzeichen hat und die Integrale rechts verschwinden. Dann muss $\left\|\pi_{i}^{k}\right\| \equiv 0 \quad$ d. h. $\quad \pi_{i .}^{k} \equiv 0 \quad$ und $\quad \mathfrak{f}=$ const gelten. Auf diese Weise lassen sich sofort einige Probleme lösen:

1) Das Minkowski-Problem für Eiflächen $\left(R_{1} R_{2}=\hat{R}_{1} \hat{R}_{2}\right)$.

2) Das Christoffel-Problem für Eiflächen $\left(R_{1}+R_{2}=\hat{R}_{1}+\hat{R}_{2}\right)$.

3) Das allgemeine Problem für Mützen mit kongruenten Rändern.

Es darf noch darauf hingewiesen werden, dass 1) auch für Eiflächen mit Ecken durch die Integralformelmethode gelöst wird [8].

b) Die Indexmethode [7], [12]. Hier untersucht man die singulären Stellen des Kurvennetzes $\sigma_{i k} d u^{i} d u^{k}=0$.

Wenn die Voraussetzungen des Hilfssatzes erfüllt sind, dann ist wegen $\left\|\pi_{i}^{k}\right\| \leqq 0$ und $K>0$ dieses Netz reell. Ziel ist hier wiederum der Nachweis $\sigma_{i k} \equiv 0 \quad$ d. h. $\quad \pi_{i .}^{k} \equiv 0$. Das geschieht in zwei Etappen.

Zunächst eine rein lokale. Man hat zweierlei zu beweisen:

a) Die singulären Stellen $\left(\sigma_{i k}=0\right)$ des Netzes sind isoliert.

$\beta$ ) Der Poincarésche Index des Netzes in einer singulären Stelle ist $\leqq 0$.

Der zweite Schritt ist jetzt von globaler Natur. Man wendet die Indexformel von Poincaré an, wonach die Summe der Indizes gleich $4(1-p)$ ist, wenn $p$ das Geschlecht der betrachteten geschlossenen orientierbaren Fläche ist. Im Fall $p=0$ liegt daher zu $\beta$ ) ein Widerspruch vor, der sich nur für $\sigma_{i k}=0$ löst.

Es lassen sich auf diese Weise sofort das Minkowski- und das ChristoffelProblem lösen. Auch das Aleksandrow-Problem ist durch diese Methode zu erfassen. Kürzlich ist von H. F. Münzner [12] auch das allgemeine Problem für Eiflächen so behandelt worden.

Freie Universität Berlin

Deutschland 


\section{Literatur}

[1] Chern, S.-s., und R. K. LAShof: On the total curvature of immersed manifolds. Amer. J. Math. 79, 1957, S. 306-318.

[2] -»- - - On the total curvature of immersed manifolds, II. - Michigan Math. J. 5, 1958, S. 5-12.

[3] Efimow, N. W.: Flächenverbiegung im Grossen. Mit einem Nachtrag von E. Rembs und K. P. Grotemeyer. - Mathematische Lehrbücher und Monographien II. VII, Akademie-Verlag, Berlin, 1957.

[4] Fenchel, W.: Über Krümmung und Windung geschlossener Raumkurven. Math. Ann. 101, 1929, S. 238-252.

[5] Grotemeyer, K. P.: Zur eindeutigen Bestimmung von Flächen durch die erste Fundamentalform. - Math. Z. 55, 1952, S. 253-268.

[6] -»- Zur Flächentheorie im Grossen: Über die Abbildung durch parallele Normalen. - Arch. Math. 9, 1958, S. 117-122.

[7] -»- Zur Flächentheorie im Grossen II: Einfache Bemerkungen zur Poincaréschen Indexmethode. - Arch. Math. 9, 1958, S. 382-388.

[8] -»- Zur Flächentheorie im Grossen III: Flächenstücke mit Kanten. - Arch. Math. 10, 1959, S. 216-220.

[9] Hsiung, C.-C.: Some uniqueness theorems on Riemannian manifolds with boundary. - Illinois J. Math. 4, 1960, S. 526--540.

[10] KuIPER, N. H.: Immersions with minimal total absolute curvature. - Colloque de géométrie différentielle globale à Bruxelles 1958, Centre Belge de Recherches Mathématiques, Librairie Universitaires / Librairie GauthierVillars, Louvain / Paris, 1959, S. 75-88.

[11] Laugwitz, D.: Differentialgeometrie. - Mathematischı Leitfäden, B. G. Teubner Verlagsgesellschaft, Stuttgart, 1960.

[12] Münzner, H. F.: Die Poincarésche Indexmethode urid ihre Anwendungen in der affinen Flächentheorie im Grossen. - Inaugural-Dissertation, Freie Universität Berlin, Berlin, 1963.

[13] SARD, A.: The measure of the critical values of differentiable maps. - Bull. Amer. Math. Soc. 48, 1942, S. 883-890.

[14] Smale, S.: A classification of immersions of the 2-sphere. - Bull. Amer. Math. Soc. 63, 1957, S. 196. 